\title{
Planned, Related or Preventable: Defining Readmissions to Capture Quality of Care
}

\author{
Leora I. Horwitz, MD, MHS $1,2,3^{*}$
}

${ }^{1}$ Division of Healthcare Delivery Science, Department of Population Health, NYU School of Medicine, New York, New York; ${ }^{2}$ Center for Healthcare Innovation and Delivery Science, NYU Langone Health, New York, New York; ${ }^{3}$ Division of General Internal Medicine and Clinical Innovation, Department of Medicine, NYU School of Medicine, New York, New York.

In this issue of the Journal of Hospital Medicine, Ellimoottil and colleagues examine characteristics of readmissions identified as planned by the planned readmission algorithm developed for the Center for Medicare \& Medicaid Services (CMS) by using Medicare claims data from 131 hospitals in Michigan. ${ }^{1}$ They found that a substantial portion of readmissions currently classified as planned by the algorithm appear to be nonelective, as defined by the presence of a charge by an emergency medicine physician or an admission type of emergent or urgent, making those hospitalizations unlikely to be planned. They suggest that the algorithm could be modified to exclude such cases from the planned designation.

To determine whether modifying the algorithm as recommended is a good idea, it is helpful to examine the origins of the existing planned readmission algorithm. The algorithm originated as a consequence of hospital accountability measures for readmissions and was developed by this author in collaboration with colleagues at Yale University and elsewhere. ${ }^{2}$ Readmission measures have been controversial in part because clearly some (undetermined) fraction of readmissions is unavoidable. Many commentators have asked that readmission measures therefore capture only avoidable or related readmissions. Avoidable readmissions are those that could have been prevented by members of the healthcare system through actions taken during or after hospitalization, such as patient counseling, communication among team members, and guideline-concordant medical care. Related readmissions are those directly stemming from the index admission. However, reliably and accurately defining such events has proven elusive. One study, for instance, found the rate of physician-assessed preventability in published studies ranged from $9 \%$ to $48 \% .^{3}$ The challenge is even greater in trying to determine preventability using just claims data, without physician review of charts. Imagine, for instance, a patient with heart failure who is readmitted with heart failure exacerbation. The readmission preceded by a

\footnotetext{
*Address for correspondence and reprint requests: Leora I. Horwitz, MD, MHS, Department of Population Health, NYU School of Medicine, 550 First Ave, TRB, Room 607, New York, NY 10016; Telephone: 646-501-2685; Fax: 646501-2706; E-mail: Leora.Horwitz@nyumc.org
}

Received May 4, 2017; Revised: July 6, 2017; Accepted: July 14, 2017

2017 Society of Hospital Medicine DOI 10.12788/jhm.2834 large fast-food meal is likely preventable; although even in this case, some would argue the healthcare system should not be held accountable for a readmission if the patient had been properly counseled about avoiding salty food. The one preceded by progressively worsening systolic function in a patient who reliably takes medications, weighs herself daily, and watches her diet is likely not. But both appear identical in claims. Related is also a difficult concept to operationalize. A recently hospitalized patient readmitted with pneumonia might have acquired it in the hospital (related) or from her grandchild 2 weeks later (unrelated). Again, both appear identical in claims.

In the ideal world, clinicians would be held accountable only for preventable readmissions. In practice, that has not proven to be possible.

Instead, the CMS readmission measures omit readmissions that are thought to be planned in advance: necessary and intentional readmissions. Defining a planned readmission is conceptually easier than defining a preventable readmission, yet even this is not always straightforward. The clearest case might be a person with a longstanding plan to have an elective surgery (say, a hip replacement) who is briefly admitted with something minor enough not to delay a subsequent admission for the scheduled surgery. Other patients are admitted with acute problems that require follow-up hospitalization (for instance, an acute myocardial infarction that requires a coronary artery bypass graft 2 weeks later). ${ }^{4}$ More ambiguous are patients who are sent home on a course of treatment with a plan for rehospitalization if it fails; for instance, a patient with gangrene is sent home on intravenous antibiotics but fails to improve and is rehospitalized for an amputation. Is that readmission planned or unplanned? Reasonable people might disagree.

Nonetheless, assuming it is desirable to at least try to identify and remove planned readmissions from measures, there are a number of ways in which one might do so. Perhaps the simplest would be to classify each hospitalization as planned or not on the UB-04 claim form. Such a process would be very feasible but also subject to gaming or coding variability. Given that there is some ambiguity and no standard about what types of readmissions are planned and that current policy provides incentives to reduce unplanned readmission rates, hospitals might vary in the cases to which they would apply such a code. This approach, therefore, has not been favored by payers to date. An alternative is to prospectively 
flag admissions that are expected to result in planned readmissions. In fiscal year 2014, the CMS implemented this option for newborns and patients with acute myocardial infarction by creating new discharge status codes of "discharged to [location] with a planned acute care hospital inpatient readmission." Institutions can flag discharges that they know at the time of discharge will be followed by a readmission, such as a newborn who requires a repeat hospitalization for repair of a congenital anomaly. ${ }^{5}$ There is no time span required for the planned readmission to qualify. However, the difficulty in broadening the applicability of this option to all discharges lies in identification and matching; there also remains a possibility for gaming. The code does not specify when the readmission is expected nor for what diagnosis or procedure. How, then, do we know if the subsequent readmission is the one anticipated? Unexpected readmissions may still occur in the interim. Conversely, what if the discharging clinicians don't know about an anticipated planned procedure? What would stop hospitals from labeling every discharge as expected to be followed by a planned readmission? These considerations have largely prevented the CMS from asking hospitals to apply the new code widely or from applying the code to identify planned readmissions.

Instead, the existing algorithm attempts to identify procedures that might be done on an elective basis and assumes readmissions with these procedures are planned if paired with a nonurgent diagnosis. Ellimoottil and colleagues attempt to verify whether this is accurate using a creative approach of seeking emergency department (ED) charges and admission type of emergent or urgent, and they found that roughly half of planned readmissions are, in fact, likely unplanned. This figure agrees closely with the original chart review validation of the algorithm. In particular, they found that some procedures, such as percutaneous cardiac interventions, appear to be paired regularly with a nonurgent principal diagnosis, such as coronary artery disease, even when done on an urgent basis.

This validation was performed prior to the availability of version 4.0 of the planned readmission algorithm, which removes several high-frequency procedures from the potentially planned readmission list (including cardiac devices and diagnostic cardiac catheterizations) that were very frequently mischaracterized as planned in the original chart validation. ${ }^{6}$ At least 8 such cases were also identified in this validation according to the table. Therefore, the misclassification rate of the current algorithm version is probably less than that reported in this article. Nonetheless, percutaneous transluminal coronary angioplasty remains on the planned procedure list in version 4.0 and appears to account for a substantial error rate, and it is likely that the authors' approach would improve the accuracy even of the newer version of the algorithm.

The advantages of the suggested modifications are that they do not require chart review and could be readily adopted by the CMS. Although seeking ED charges for Medicare is somewhat cumbersome in that they are recorded in a different data set than the inpatient hospitalizations, there is no absolute barrier to adding this step to the algorithm, and doing so has substantial face validity. That said, identifying ED visits is not straightforward because nonemergency services can be provided in the ED (ie, critical care or observation care) and because facilities and providers have different billing requirements, producing different estimates depending on the data set used. ${ }^{7}$ Including admission type would be easier, but it would be less conservative and likely less accurate, as this field has not been validated and is not typically audited. Nonetheless, adding the presence of ED charges seems likely to improve the accuracy of the algorithm. As the CMS continues to refine the planned readmission algorithm, these proposed changes would be very reasonable to study with chart validation and, if valid, to consider adopting.

Disclosure: Dr. Horwitz reports grants from Center for Medicare \& Medicaid Services, grants from Agency for Healthcare Research and Quality, during the conduct of the study.

\section{References}

1. Ellimoottil C, Khouri R, Dhir A, Hou H, Miller D, Dupree J. An opportunity to improve Medicare's planned readmissions measure. J Hosp Med. 2017;12(10): $840-842$.

2. Horwitz LI, Grady JN, Cohen DB, et al. Development and validation of an algorithm to identify planned readmissions from claims data. J Hosp Med. 2015;10(10):670-677.

3. Benbassat J, Taragin M. Hospital readmissions as a measure of quality of health care: advantages and limitations. Arch Intern Med. 2000;160(8):1074-1081.

4. Assmann A, Boeken U, Akhyari P, Lichtenberg A. Appropriate timing of coronary artery bypass grafting after acute myocardial infarction. Thorac Cardiovasc Surg. 2012;60(7):446-451.

5. Inpatient Prospective Payment System/Long-Term Care Hospital (IPPS/LTCH) Final Rule, 78 Fed. Reg. 27520 (Aug 19, 2013) (to be codified at 42 C.F.R. Parts 424, 414, 419, 424, 482, 485 and 489). http://www.gpo.gov/fdsys/pkg/FR-2013-0819/pdf/2013-18956.pdf. Accessed on May 4, 2017.

6. Yale New Haven Health Services Corporation Center for Outcomes Research and Evaluation. 2016 Condition-Specific Measures Updates and Specifications Report: Hospital-Level 30-Day Risk-Standardized Readmission Measures. March 2016.

7. Venkatesh AK, Mei H, Kocher KE, et al. Identification of emergency department visits in Medicare administrative claims: approaches and implications. Acad Emerg Med. 2017;24(4):422-431. 